\title{
Shrinkage of Dynamesh IPOM mesh in 6-week follow-up - an experimental study
}

\author{
Andrzej Jamry ${ }^{1}$, Marek Jałyński ${ }^{2}$ Janusz Śmigielski ${ }^{3}$, Marian Brocki ${ }^{4}$ \\ $12^{\text {nd }}$ Department of Surgery, Hospital in Starachowice, Poland \\ 2Department of Surgery and Roentgenology, University of Warmia and Mazury, Olsztyn, Poland \\ 3Department of Information and Statistics, Medical University, Lodz, Poland \\ ${ }^{4}$ Department of Thoracic Surgery, Medical University, Lodz, Poland
}

Videosurgery and other miniinvasive techniques 2011; 6 (1): 19-23 DOI: $10.5114 /$ wiitm.2011.20988

\begin{abstract}
Introduction: The process of shrinkage of a mesh in the peritoneal cavity is not fully understood, but it may cause reduction of the implant area down to 30-50\%. Therefore, knowledge on the magnitude of the phenomenon for the used prosthesis enables proper sizing. This reduces the relapse rate on the one hand and the use of excess material, being a "foreign body", on the other hand.

Aim: 1. To assess shrinkage of Dynamesh IPOM (intraperitoneal onlay mesh) implanted into the peritoneal cavity in 6-week follow-up. 2. To assess whether the type of slow-absorbable suture used to attach the prosthesis affects the process.

Material and methods: The study was conducted in an animal model (swine). In each one of 12 animals two mesh fragments were implanted. In each case, one fragment was sutured with PDS II and the other one with Maxon. After 6 weeks, mesh area was measured and compared with respective baseline values, calculating linear shortening and area loss. The second part of the analysis was addressed to determine the effect of the used suture on the phenomenon. To do this, mesh linear and area loss was calculated for both groups (PDS and Maxon), and the results were compared with Mann-Whitney $U$ test.

Results and conclusion: 1. Dynamesh IPOM demonstrates linear shortening by $12.53 \%$ and area loss of $23.85 \%$ after 6 weeks. These values are significant and should be accounted for during implant sizing. 2. There was no statistically significant difference in shrinkage parameters in the groups with meshes attached with PDS I/ and Maxon.
\end{abstract}

Key words: shrinkage, surgical mesh, laparoscopy, ventral hernia, cicatrix.

\section{Introduction}

The process of mesh shrinkage and its sequelae after laparoscopic hernia repair are not completely understood. The process results from physiological scar maturation, leading to a decrease of water content, rather than shrinkage of the prosthesis itself, as it used to be explained. The magnitude of it is related to inflammatory activity and foreign body reaction, which are mediated by the structure and material of the used mesh, type of fixatives, collagen disorders and environmental factors.

This phenomenon may cause a decrease of scar area around the prosthesis of up to $30-50 \%$. This causes relapses, tension at fixation points with subsequent chronic pain, and "button-like" hernias and haematomas. Therefore, to avoid the mesh becoming smaller than the repaired defect, it is recommended 
to use prostheses larger by $5 \mathrm{~cm}$ than the defect diameter. Such a created overlap additionally stabilizes the repair thanks to intra-abdominal pressure action on its surface.

However, excess foreign material may promote an extensive inflammatory reaction, neuralgia, "foreign body" sensations, adhesions and eventually erosions to the bowel lumen. Therefore, knowledge of the magnitude of the process precipitated by the type of used material is necessary to accurately size the prosthesis. Unfortunately, reports on the matter are discordant. Thus the current study aims to assess the phenomenon for Dynamesh IPOM mesh 6 weeks after implantation.

The body's response to implant fixation material may be an additional factor influencing scar formation. Therefore the secondary goal was to evaluate whether the type of used fixation suture affects shrinkage of the prosthesis [1-15].

\section{Aim}

1. To evaluate shrinkage of Dynamesh IPOM mesh 6 weeks after implantation into the peritoneal cavity.

2. To assess whether the type of slow-absorbable suture (PDS II or Maxon) used for fixation affects the shrinkage process.

\section{Material and methods}

Dynamesh IPOM, a two-layered mesh, consisting of a parietal layer made from polypropylene monofilament thread with large pores, durability of $36 \mathrm{~N} / \mathrm{m}$ and density of $108 \mathrm{~g} / \mathrm{m}^{2}$, and a visceral layer made from polyvinylidene fluoride (PVDF), was used in the study.

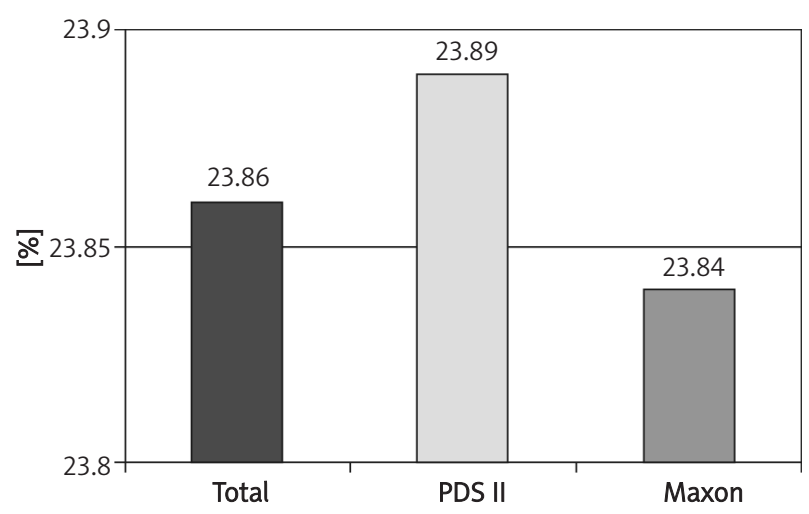

Figure 1. Mesh surface area loss (total and fixated with PDS II and Maxon)
The study was conducted on an animal model (Resolution 02/2009 of the Ethical Committee of the University of Warmia and Mazury) -12 pigs weighing between $30 \mathrm{~kg}$ and $40 \mathrm{~kg}$. Each specimen had implanted two mesh fragments, measuring $6.0 \times 6.6 \mathrm{~cm}$. One fragment was fixated with PDS II 1-0 suture, and the other with Maxon 1-0 suture. Antimicrobial prophylaxis was administered $30 \mathrm{~min}$ before the procedure.

Under general anaesthesia, an $11 \mathrm{~mm}$ optical trocar was inserted into the peritoneal cavity in the medial line, after creating pneumoperitoneum with a Veress needle, using $12 \mathrm{mmHg}$ pressure. Then, a $5 \mathrm{~mm}$ working trocar was inserted about $4-5 \mathrm{~cm}$ caudally from the optical system. Through the $11 \mathrm{~mm}$ port two mesh fragments with four pre-attached fixating sutures at $4 \mathrm{~mm}$ intervals were sequentially inserted. One of the fragments was equipped with PDS II sutures, and the other with Maxon sutures. The animals were bred in a routine manner. After 6 weeks, both meshes were measured in two dimensions; for all meshes, the sum of the length and width measurements and surface area were calculated. The results were compared to baseline values to yield linear shortening and area loss.

The second part of the analysis was to determine the effect of suture type on the process examined. To do this, linear shortening and area loss of all meshes in PDS and Maxon groups were calculated; the differences were analysed statistically with the MannWhitney $\mathrm{U}$ test.

\section{Results}

Six weeks after surgery the sum of the length and width of all meshes was $264.5 \mathrm{~cm}$, and when compared to the baseline value of $302.4 \mathrm{~cm}$, there was a decrease of $37.9 \mathrm{~cm}(12.53 \%)$. Total surface area was $723.59 \mathrm{~cm}^{2}$, and when compared to the baseline value of $950.4 \mathrm{~cm}^{2}$, a decrease of $228.81 \mathrm{~cm}^{2}$ (23.86\%) was found (Figure 1 ).

There was a linear loss of meshes fixated with PDS II from 151.2 to $131.8 \mathrm{~cm}$ (12.8\%), and from 151.2 to $132.7 \mathrm{~cm}$ (12.24\%) for Maxon. Mesh surface area when fixated with PDS II decreased from $475.2 \mathrm{~cm}^{2}$ to $361.9 \mathrm{~cm}^{2}(23.89 \%)$ and from $475.2 \mathrm{~cm}^{2}$ to $361.9 \mathrm{~cm}^{2}$ (23.84\%) when fixated with Maxon. The difference was tested with the Mann-Whitney U test; no statistical significance was found.

Additional analysis of distribution of surface area loss was as follows: shortening by $0-10 \%$ 
occurred in $37.5 \%$, by $11-20 \%$ in $50 \%$, by $20-25 \%$ in $8.3 \%$, and more than $25 \%$ in $4.2 \%$ of the studied prosthesis fragments. The conclusion is that most (87.5\%) meshes shrank by no more than $20 \%$ after 6 weeks.

\section{Discussion}

The available data on prosthesis shortening have been very discordant. The results appear to result from usage of meshes of various material and structure, experimenting with various animal models, different observation times and various assessment methods (e.g. measurement of only one dimension or surface area analysis). The results of experimental studies are presented in Table I.

The above summary illustrates very high variation, even in studies on the same animal species with the same observation period, which would suggest that the type of used prosthesis accounted for the differences. However, inconsistency of results for the same mesh types (as summarized in Table II) proves this hypothesis wrong.

Thus some authors claim that the relation between the used material and the inflammatory response is not clearly defined and remains uncertain. The analysis of available data is hindered because ongoing trials on prostheses of older types are interrupted and new products are studied instead. To summarize, based on the published data from experimental studies, mean surface area loss was $38 \pm 6 \%$.

The results of the current study, as opposed to the above-mentioned value, showed that the area of Dynamesh IPOM decreased by less than $24 \%$, which may indicate that the magnitude of the shrinkage process for this mesh is below average. However, the above reservations apply to this conclusion [4, 7-10, 15-17].

There are more concerns on translation of experimental data to the extent of the process in humans. Mesh shortening was originally observed during repeat surgery of patients who had implanted meshes. This group is small, and the reasons are obvious. On the other hand, there is no possibility for noninvasive shortening measurement, as most of the used prostheses are invisible in routinely available imaging studies. Only two reports provide data obtained from imaging studies.
Table I. Summary of data from experimental studies

\begin{tabular}{|lcc|}
\hline Animal species & $\begin{array}{c}\text { Observation time } \\
\text { [weeks] }\end{array}$ & $\begin{array}{c}\text { Mesh shortening } \\
{[\%]}\end{array}$ \\
\hline Rabbit & 12 & 7.6 \\
\hline Rabbit & 52 & 32 \\
\hline Rabbit & 16 & 34.7 \\
\hline Rabbit & 12 & 41 \\
\hline Rat & 4 & 44.2 \\
\hline Pig & 12 & 43.5 \\
\hline Pig & 4 & 37 \\
\hline Rabbit & 21 & 50.8 \\
\hline
\end{tabular}

Table II. Comparison of shortening of various mesh types

\begin{tabular}{|lc|}
\hline Material & Mesh shortening [\%] \\
\hline Proceed & $11-44$ \\
\hline Supramesh & $7-38$ \\
\hline Parietex & $15-29.5$ \\
\hline ePTFE & $7.6-50.8$ \\
\hline Dynamesh & $12.6-24$ \\
\hline PPM & 38 \\
\hline
\end{tabular}

The first of the two was conducted on 40 patients and the follow-up period was 17 months. The Aquarius Net system was used to calculate linear loss of a dual-mesh prosthesis and a $7.5 \%$ loss was found. In the other study, polypropylene meshes with marked edges were used, which enabled linear loss assessment based on imaging studies. The authors reported shortening of $6.2 \%$ using this technique. Both these results are lower by almost half than the result from the current experimental study (more that 12\%) and most other experimental studies. Therefore, the suggestion of a different reaction to a foreign body in different species persists. However, the groups were relatively small and there are only two reports, which indicates the need for further studies in the field [5, 8 , 10, 13, 15, 17-19].

Follow-up period might be another factor influencing the obtained results. There are available data suggesting that the scarring process is most exten- 
sive in the acute inflammatory phase during the first 6 weeks and is not significantly different than when examined after 6 months. However, considering the spread of the published data, scar remodelling in later periods cannot be ruled out, with consequences such as mesh area loss. The period in the current study was 6 weeks, which was also conditional on the assumptions of another study, running in parallel $[5,8,10,13,15,17-19]$.

Additional analysis of mesh shortening distribution was also performed. In human studies there was no mesh shortening in $28 \%$ of patients, whereas there was shortening by $1-10 \%$ in $40 \%$ of cases, by $10-20 \%$ in $25 \%$, and more than $25 \%$ in $7 \%$. The authors of the above-mentioned study conclude, based on these results, that individual tendencies have a major effect on the magnitude of the analysed phenomenon. In the current study all meshes shortened, while the dimensions of most (87.5\%) dropped by less than $20 \%$ within 6 weeks. However, there were significant differences between individual specimens, which would support the hypothesis of individual tendencies for extent of scarring in response to a foreign body.

It is known that scarring resulting from reaction to a foreign body is responsible for mesh shortening. Therefore, implant fixation may also influence the process. In studies on the use of sutures and ProTacks there was prosthesis shortening by $5.8 \%$ and $6.2 \%$, respectively. However, in the current comparison of two slow-absorbable sutures (PDS II and Maxon) no significant difference in surface area loss after 6 weeks was found. The above data suggest that type and material of fixation have no significant effect on the mesh shortening process $[2,5,7,15,20]$.

\section{Conclusions}

Like most implants for hernia repair, Dynamesh IPOM meshes are subject to shrinkage, with surface area loss of above $23 \%$ after 6 weeks. This is a significant value, and should be taken into account when sizing the implant during surgery. The performed analysis of slow-absorbable sutures (PDS II and Maxon) showed no statistically significant effect of suture type on prosthesis shrinkage.

\section{References}

1. Vavrik J, Foltynova V, Vitkova I, et al. Changes in abdominal wall after mesh implantation in rats. Med Sci Monit 2000; 6: 476-9.
2. Korencov M, Sauerland S, Arndt M. Randoimzed clinical trial of suture repair polypropylene mesh or autodermal hernioplasty for incisional hernia. Br J Surg 2002; 89: 50-6.

3. Leber GE, Garb JL, Alexander Al, et al. Long-therm complications associated with prosthetic repair of incisional hernias. Arch Surg 1998; 133: 378-82.

4. Schug-Pa BC, Tamme C, Sommerer F, et al. Composite meshes for laparoscopic intraperitoneal repair of abdominal wall hernias comparison of biocompatibility in an experimental study using the porcine model. 10 $0^{\text {th }}$ World Congress of Endoscopic Surgery 2006 Berlin. Abstract book 0036.

5. Wagner M, Lutz A, Bruegger E, et al. Mesh shrinking after laproscopic ventral hernia repair a prospective study. 10th World Congress of Endoscopic Surgery 2006 Berlin. Abstract book 0042.

6. LeBlank K. Laparoscopic incisional hernia repair; are transfascial sutures necessary? A review of literature. Surg Endosc 2007; 21: 508-13.

7. Klosterhalfen B, Junge K, Klinge U. The lightweight and large porous mesh concept for hernia repair. Expert Rev Med Devices 2005; 2: 103-17.

8. Klinge U, Klosterhalfen B, Ottinger AP. PVDF as new polymer for the construction of surgical meshes. Biomaterials 2002; 23: 3487-93.

9. Farrakha M. Laparoscopic treatment of ventral hernia. A bilayer repair. Surg Endosc 2000; 14: 1156-8.

10. Jacob BP, Hogle NJ, Durak E, et al. Tissue ingrowth and bowel adhesion formation in an animal comparative study; popypropylene versus Proceed versus Parietex Composite. Surg Endosc 2007; 4: 629-33.

11. Meissner K, Jirikowski B, Szecsi T. Repair of parietal hernia by overlapping onlay reinforcement or gap bringing replacement polypropylene mesh; preliminary results. Hernia 2000; 4: 29-32.

12. Mesh JJ. Shrinking and consequences for operation tactics in laproscopic incisional hernia repair. Hernia 2009; 121 (Suppl. 1): S33-S63.

13. Gonzales R, FugateK, Mc Clusky D, et al. Relationship between tissue ingrowths and mesh contraction World J Surg 2005; 29: 1038-43.

14. Benchetrit S, Debaert M, Dufilho A, et al. Laproscopic and open abdominal wall reconstruction using Parietex meshes. Clinical results in 2700 hernias. Hernia 1998; 2: 57-62.

15. Malman L, Jenkins E, Hamilton N, et al. Evaluation of crosslinked and non-crosslinked biologic mesh in porcine model of ventral hernia repair. Hernia 2009; 13 (Suppl. 1): S5-15.

16. Fowler D. Tissue ingrowth and bowel adhesion formation animal comparative study ePTFE vs. ePTFE - Polypropylene vs. Parietex composite. Advence on parietal repair "guideline in surgery". $26^{\text {th }}$ International Congress of the European Hernia Society 2004 Prague.

17. Ramshaw B, Abiad F, Voeller G, et al. Polyester (Parietex) mesh for total extraperitoneal laparoscopic inguinal hernia repair: initial experience in the United States. Surg Endosc 2003; 17: 498-501

18. Majercik S, Tsikitis V, Iannitti A. Strength of tissue attachment to mesh after ventral hernia repair with synthetic composite mesh in a porcine model. Surg Endosc 2006; 20: 1671-74. 
19. Tang CN, Tsui DKK, Ha JPY, et al. Laparoscopic repair of incisional hernia a randomized trial. 14th International Congress of the European Association for Endoscopic Surgery (EAES). Abstract book 2006; 029.

20. Czudek S, Skrovina M, Adamcik L. Laparoscopic treatment of inguinal hernia - TOM (transabdominal onlay mesh). Videosurgery and other miniinvasive techniques 2009; 4: 16-9. 